\title{
Development of a Video-Microscopic Tool To Evaluate the Precipitation Kinetics of Poorly Water Soluble Drugs: A Case Study with Tadalafil and HPMC
}

Christfort, Juliane Fjelrad; Plum, Jakob; Madsen, Cecilie Maria; Nielsen, Line Hagner; Sandau, Martin; Andersen, Klaus; Müllertz, Anette; Rades, Thomas

Published in:

Molecular Pharmaceutics

Link to article, DOI:

10.1021/acs.molpharmaceut.7b00422

Publication date:

2017

Document Version

Peer reviewed version

Link back to DTU Orbit

Citation (APA):

Christfort, J. F., Plum, J., Madsen, C. M., Nielsen, L. H., Sandau, M., Andersen, K., Müllertz, A., \& Rades, T. (2017). Development of a Video-Microscopic Tool To Evaluate the Precipitation Kinetics of Poorly Water Soluble Drugs: A Case Study with Tadalafil and HPMC. Molecular Pharmaceutics, 14(12), 4154-4160.

https://doi.org/10.1021/acs.molpharmaceut.7b00422

\section{General rights}

Copyright and moral rights for the publications made accessible in the public portal are retained by the authors and/or other copyright owners and it is a condition of accessing publications that users recognise and abide by the legal requirements associated with these rights.

- Users may download and print one copy of any publication from the public portal for the purpose of private study or research.

- You may not further distribute the material or use it for any profit-making activity or commercial gain

- You may freely distribute the URL identifying the publication in the public portal 


\section{Development of a Video-Microscopic Tool to Evaluate the 2 Precipitation Kinetics of Poorly-Water Soluble Drugs: A Case Study 3 with Tadalafil and HPMC}

4 Juliane Fjelrad Christfort ${ }^{1 *}$, Jakob Plum ${ }^{1 *}$, Cecilie Maria Madsen ${ }^{1, \dagger}$, Line Hagner Nielsen ${ }^{2}$, Martin

5 Sandau $^{3}$, Klaus Andersen ${ }^{3}$, Anette Müllertz ${ }^{1,4, \ddagger}$, Thomas Rades ${ }^{1,5}$

6 1) Department of Pharmacy, University of Copenhagen, DK-2100 Copenhagen, Denmark

7 2) Department of Micro and Nanotechnology, Technical University of Denmark, DK-2800 Lyngby, Denmark

8 3) Philips BioCell A/S, DK-3450 Allerød, Denmark

9 4) Bioneer:FARMA, University of Copenhagen, DK-2100 Copenhagen, Denmark

10 5) Faculty of Science and Engineering, Åbo Akademi University, Tykistökatu 6A, 20521 Turku, Finland

11 *These authors contributed equally to the manuscript.

12

13

Keywords

supersaturation, precipitation, oral drug delivery, crystalline

15

16

Graphical abstract

17

18

19

20

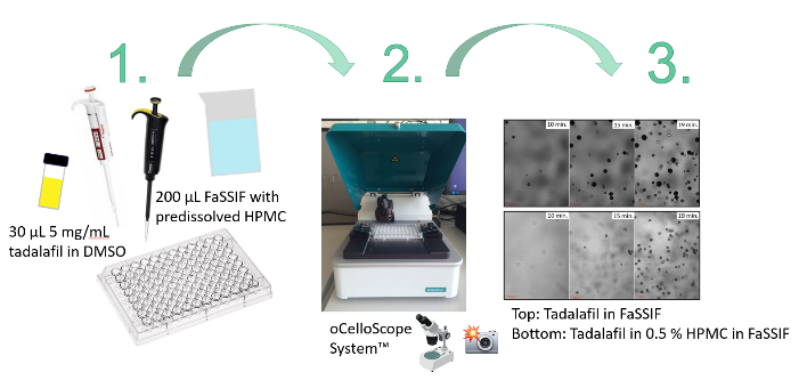

\footnotetext{
† Currently employed at Analytical Research and Development, H. Lundbeck A/S, Ottiliavej 9, Valby, Denmark

‡ Correspondence to: Anette Müllertz

Department of Pharmacy,

University of Copenhagen,

Universitetsparken 2

DK-2100 Copenhagen, Denmark

Telephone: 0045 35336440; Fax: 004535336001.

E-mail address: anette.mullertz@sund.ku.dk
} 
22 Many drug candidates today have a low aqueous solubility, and hence may show a low oral 23 bioavailability, presenting a major formulation and drug delivery challenge. One way to increase the 24 bioavailability of these drugs is to use a supersaturating drug delivery strategy. The aim of this study 25 was to develop a video-microscopic method, to evaluate the effect of a precipitation inhibitor on 26 supersaturated solutions of the poorly soluble drug tadalafil, using a novel video microscopic small scale setup. Based on preliminary studies, a degree of supersaturation of 29 was chosen for the supersaturation studies with tadalafil in FaSSIF. Different amounts of HPMC were predissolved in FaSSIF to give four different concentrations, and the supersaturated system was then created using a solvent shift method. Precipitation of tadalafil from the supersaturated solutions was monitored by video-microscopy as a function of time. Single-particle analysis was possible using commercially available software, however, to investigate the entire population of precipitating particles (i.e. their number and area covered in the field of view) an image analysis algorithm was developed (multiparticle analysis). The induction time for precipitation of tadalafil in FaSSIF was significantly prolonged by adding $0.01 \%(\mathrm{w} / \mathrm{v})$ HPMC to FaSSIF, and the maximum inhibition was reached at 0.1 \% (w/v) HPMC, after which additional HPMC did not further increase the induction time. The singleand multi-particle analyses yielded the same ranking of the HPMC concentrations, regarding the inhibitory effect on precipitation. The developed small scale method to assess the effect of precipitation inhibitors can speed up the process of choosing the right precipitation inhibitor and the concentration to be used.

\section{Introduction}

It has been estimated that approximately 75 - $90 \%$ of low molecular weight drug candidates in development can be considered poorly soluble in water (1-3), often leading to low and inconsistent bioavailability. Therefore, there is a growing need for formulation strategies enabling increased oral bioavailability. One such strategy is the use of supersaturating drug delivery systems which can induce supersaturation, e.g. in the small intestine $(4,5)$.

In a supersaturated system, the drug concentration exceeds the equilibrium solubility of the drug thus, increasing the free drug concentration available for absorption in the small intestine. According to Fick's law, an increased free drug concentration will lead to a higher flux and hence, a higher absorption. A common way to describe a supersaturated system is to use the degree of supersaturation (DS). DS is defined as the ratio between the concentration in the supersaturated solution and the thermodynamic equilibrium solubility of the drug in the same medium (6): 
Supersaturated systems are thermodynamically unstable and will inevitably be driven towards equilibrium solubility over time, which poses a significant challenge for the formulation work using such systems (4). In a supersaturated solution, the equilibrium will be restored by precipitation of the drug, which will, consequently, lower the free drug concentration available for absorption. The precipitation process can be split into two sub-processes, having their own kinetics, i.e. nucleation and crystal growth (7). Nucleation happens when molecules interact to form a cluster of a critical radius. This critical cluster formation requires a specific amount of energy. Once a nucleus has been formed and starts to grow, the process is irreversible and crystal growth occurs. Nucleation occurs prior to crystal growth; however, nucleation continues to occur following the formation of the first nucleus making it difficult to distinguish the nucleation and crystal growth kinetics from each other (7). The induction time (tind), being the time point at which the first precipitate is formed, is often used as a proxy to describe the nucleation process. In case of a supersaturating drug delivery strategy, it is desired to prolong the time until precipitation, and hereby, the duration of the existence of a supersaturated system. This can be achieved by introducing an excipient to reduce or inhibit precipitation, a so called precipitation inhibitor (PI) (4). Polyvinylpyrrolidone (PVP) (8), (hydroxypropyl)methyl cellulose (HPMC) (9), and cyclodextrins such as sulfobutyl-ether $\beta$-cyclodextrin (10) are commonly used as PIs. The PIs act either by decreasing nucleation or crystal growth rate (or both) through various pathways (4). HPMC is often used as a PI in pharmaceutical studies (6, 10-12) and it has previously been shown to delay the onset of precipitation (i.e. to prolong tind) of different drugs such as tadalafil (6), dapivirine (10), and RS-8359 (13). Several mechanisms of action have been proposed; the effect of HPMC may be due to hydrogen-bonding between the polymer and the drug (this would lead to a reduction in the nucleation kinetics) $(9,14)$, or adsorption of HPMC to the crystal surface of a growing crystal (this would reduce the crystal growth kinetics) (15). HPMC and other polymers may also work as precipitation inhibitors by increasing the viscosity of the dissolution medium, hereby reducing molecular mobility and diffusion coefficient of the precipitating drug (4). Addition of HPMC to the dissolution medium has also been shown to change the crystal structure of the precipitate of several drugs compared to a PI-free system (16), further indicating the presence of molecular interactions between the drug and the polymer during the precipitation process. It is generally known that the effect of a PI is very drug specific, and the optimal type and concentration of polymer to inhibit precipitation of a supersaturated solution will vary, depending on the drug of interest $(8,12,17)$. Warren et al. (12) investigated combinations of 42 polymers and three drugs and found very different effects on the same drug depending on polymer type, concentration and grade used as PI. Hence, it will be necessary to test several polymers in different concentrations before choosing the optimal PI type, concentration and grade for a given drug, and therefore a high-throughput, small scale setup to study precipitation kinetics is highly needed.

Precipitation studies are conventionally performed in a regular dissolution setup where the drug concentration is monitored over time (18), and the detection of precipitation is often based on a decrease in drug concentration in solution and not on the formation of particles. A direct way of studying precipitation is the use of microscopic methods. The advantage of a microscopic approach is the direct visualization of the precipitate, instead of the use of a surrogate marker (concentration of dissolved drug using e.g. in situ UV measurements). This gives the opportunity to investigate the early stages of precipitation where the amount of precipitate is not sufficient for a baseline shift due to light scattering in the visible range of the electromagnetic spectrum.

Previously, microscopy has been used to investigate precipitation in supersaturated gels over a period of days (9). Conventional microscopy, however, is time consuming and labour intensive, and therefore more automated methods have been developed for different purposes. For example, Wu et al. (19) have developed a method for online monitoring of nucleation and crystal growth using polarized light microscopy and subsequent image analysis, and recently, a method describing single pellet dissolution in a 24-well plate using video-microscopy has been published, and it was found that this method had an excellent correlation to UV measurements (20).

The combination of conventional microscopy and video analysis has furthermore been used for investigations of bacterial growth (21), in a commercially available 96-well plate setup called the oCelloScope ${ }^{\mathrm{TM}}$, specifically developed for this purpose. The aim of the current study was to develop a video-microscopic tool to screen for the effect of PIs for early drug development using this equipment. The model systems consisted of supersaturated solutions of the poorly-water soluble drug tadalafil and $\mathrm{HPMC}$ as PI.

\section{Materials and Methods}

101

\subsection{Materials}

Fasted state simulated intestinal fluid (FaSSIF) powder was purchased from biorelevant.com (London, UK). Tadalafil was purchased from AK Scientific (Union City, Ca, USA). Sodium chloride, monobasic sodium phosphate dihydrate and dimethyl 
sulfoxide (DMSO) were acquired from Merck Millipore (Darmstadt, Germany). Sodium hydroxide was obtained from VWR Chemicals (Leuven, Belgium) and hydroxypropyl methyl cellulose (HPMC, Pharmacoat 6066 cp) was purchased from Shin Etsu (Tokyo, Japan). Nunc 96-well microplates with Nunclon Delta Surface were obtained from Thermo Fisher Scientific (Waltham, MA, USA). Q-max RR syringe filters were acquired from Frisenette (Knebel, Denmark).

109

\subsection{Methods}

\subsubsection{Equilibrium solubility of tadalafil}

The solubility of tadalafil in FaSSIF + $13 \%$ (v/v) DMSO and FaSSIF + $13 \%$ (v/v) DMSO + HPMC in increasing concentrations were investigated using a shake-flask method at room temperature. FaSSIF containing $13 \%$ (v/v) DMSO was prepared by adding $1.5 \mathrm{~mL}$ DMSO to $10 \mathrm{~mL}$ of FaSSIF. Then excess tadalafil ( $>10 \mathrm{x}$ equilibrium solubility) was added. The suspension was shaken for $24 \mathrm{~h}$ and samples were taken at three time points during the experiment $(3 \mathrm{~h}, 22 \mathrm{~h}$ and $24 \mathrm{~h}$ after starting the experiment). The samples were filtered through a $0.22 \mu \mathrm{m}$ PTFE Hydrophilic filter (Q-Max RR Syringe Filter) prior to quantification by high-performance liquid chromatography (HPLC). All experiments were performed in triplicate. Analysis was performed on a C18 column (Kinetix XB-C18, $5 \mu \mathrm{m}$, Phenomenex, (Torrance, California, U.S)) with a mobile phase of $0.1 \%$ (v/v) trifluoroacetic acid in 40:60 acetonitrile:water (v/v) in a Dionex HPLC with UV detection at $284 \mathrm{~nm}$.

\subsubsection{Preparation of a supersaturated tadalafil solution in FaSSIF}

FaSSIF was prepared according to the instructions of the manufacturer and HPMC $(0.0-0.5 \%$ w/v $)$ was added. The mix was stirred overnight in order for the polymer to equilibrate in the medium (22).

The studies were conducted in 96-well microplates at room temperature by spiking $30 \mu \mathrm{L}$ of a tadalafil-DMSO stock solution (5 $\mathrm{mg} / \mathrm{mL}$ ) into $200 \mu \mathrm{L}$ FaSSIF with or without predissolved HPMC, to induce a supersaturated tadalafil solution with a concentration of $652 \mu \mathrm{g} / \mathrm{mL}$ (DS = 29). The solution was mixed by pipetting up and down two times when spiking tadalafil into the FaSSIF filled well. The molecular weight of HPMC was $36 \mathrm{kDa}(23)$ and the molar drug:polymer ratio spanned from 14-7030.

\subsection{3 oCelloScope ${ }^{\mathrm{TM}}$ setup}

The precipitation studies were performed on an oCelloScope System ${ }^{\mathrm{TM}}$ (Philips Biocell A/S, Allerød, Denmark). This system is a video-microscopic setup creating a series of micrographs using time-lapse technology, as described by Fredborg et al. (21). The experiments were performed in 24 replicates for each HPMC concentration. For obtaining 24 replicates in total, 3 or 4 experiments were run simultaneously 8 or 6 times. The illumination level and focus were set manually and adjusted individually before each experiment to ensure high micrograph quality for the subsequent image analysis. The illumination level interval varied from 300400 and the focus from 2800-2990 depending on the experiment, and the illumination time was kept constant at 2 ms throughout all experiments. Twenty micrographs were acquired of each well at every time point creating an 855 um length of the scan and thereby, covering $1.49 \mathrm{~mm}^{2}$ of the well. The number of repetitions and the repetition intervals were chosen depending on the concentration of HPMC, and designed to cover a period of $22 \mathrm{~min}$. Around the tind, micrographs were acquired every $20 \mathrm{~s}$.

\subsubsection{Single-particle analysis}

Micrographs acquired by the oCelloScope ${ }^{\mathrm{TM}}$ were analysed with the UniExplorer software version 6.0, accompanying the equipment (Philips Biocell A/S, Allerød, Denmark). Using the segmentation tool in UniExplorer software, the areas of the individual particles for every measurement time point were calculated. The tind was determined as the time point where the software identified the first particle in focus. Crystal growth was determined from the area of one well-defined particle, often the first particle to appear, calculated by the software. The particle areas were exported and further data treatment was performed using Excel 2010 (Microsoft Office, Wa, USA). Visualization and statistical analysis were performed using GraphPad Prism version 6.0 (GraphPad software Inc. Ca, USA). Statistical differences were determined using one-way ANOVA with a Sidak multiple comparison test.

\subsubsection{Multi-particle analysis}

In contrast to the single-particle analysis, multi-particle analysis determined crystal growth from the area of all the particles precipitating within the field of view. The areas covered by particles were segmented using UniExplorer's gradient segmentation algorithm and the H-minima Transform algorithm (24). From the segmented areas, crystal growth was then quantified as the percentage of the total micrograph area covered by the particles and as the number of particles. The number of particles was counted automatically using the two stage circular Hough transform (25). With this approach circles were fitted to the segmented areas, where the number of fitted circles corresponded to the number of particles in the micrograph. 


\section{Results and Discussion}

3.1 Solubility, micrograph quality and morphology

The equilibrium solubility of tadalafil was investigated in FaSSIF containing $13 \%$ (v/v) DMSO with and without addition of up to $1 \%(\mathrm{w} / \mathrm{v})$ HPMC. The solubility was found to be $22.5 \pm 1.6 \mu \mathrm{g} / \mathrm{mL}$, and addition of HPMC did not increase the solubility significantly ( $\mathrm{p}>0.05)$.

The precipitation kinetics of the supersaturated tadalafil solutions were monitored using the oCelloScope ${ }^{\mathrm{TM}}$ set-up. Figure $1 \mathrm{~A}-\mathrm{C}$ shows micrographs of pure FaSSIF, a precipitated tadalafil suspension in FaSSIF and a precipitated tadalafil suspension in FaSSIF with $0.5 \%$ (w/v) HPMC after 14 min, respectively. The presence of $0.5 \%(\mathrm{w} / \mathrm{v})$ HPMC clearly decreased the number of precipitated particles in the frame observed after 14 min compared to the tadalafil suspension in FaSSIF. The difference in background colour is due to different illumination settings. Pure FaSSIF (Figure 1A) contained silhouettes of larger structures of approximately $100 \mu \mathrm{m}$, however these silhouettes in pure FaSSIF were clearly distinguishable from the precipitate of tadalafil in FaSSIF. The darker blurry areas of the micrographs in Figure $1 \mathrm{~B}$ and $\mathrm{C}$ were most likely precipitated particles out of focus.

The precipitate in pure FaSSIF (orange arrow, Figure 1B) had a round and compact core with a less dense outer layer (Figure 1B), whereas the precipitate in FaSSIF with $0.5 \%$ (w/v) HPMC (blue arrow, Figure 1C) appeared less dense and less regular. However, later appearing particles in pure FaSSIF (green arrow, Figure 1B) were similar to the less dense particles found in FaSSIF with HPMC.

Tadalafil has previously been shown to precipitate in a crystalline form (6), and similar differences in precipitate morphology have been observed for other compounds (7). For studying the morphology of the precipitate, Lindfors et al. (7) prepared supersaturated solutions of bicalutamide, let them stand for a minimum of $72 \mathrm{~h}$ to precipitate before taking images to analyse morphology and number of particles manually. Crystallization of the supersaturated bicalutamide system without PVP led to a star-like morphology of the particles, whereas more compact and spherical particles were formed in the presence of $0.01 \%$ (w/w) PVP as PI (7). Furthermore, Lindfors et al. (7) reported morphology differences in precipitate particles formed without PI present dependent on the age of the particles, similar to the precipitate morphology observed for tadalafil (Figure 1B).
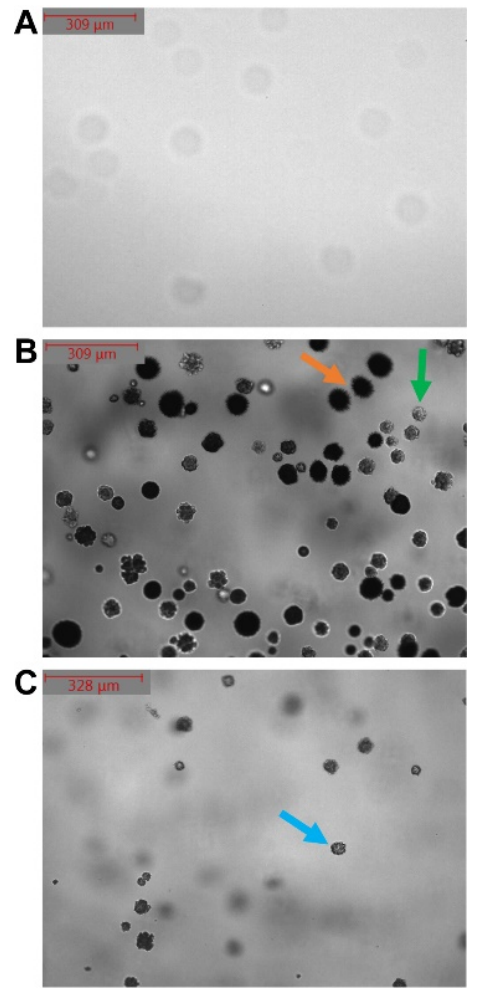

Figure 1. Micrographs from the oCelloScope ${ }^{\mathrm{TM}}$. A: pure FaSSIF, B: $652 \mu \mathrm{g} / \mathrm{mL}$ tadalafil in FaSSIF (t: $\left.14.1 \mathrm{~min}\right)$, C: $652 \mu \mathrm{g} / \mathrm{ml}$ tadalafil in FaSSIF with $0.5 \%$ (w/v) HPMC (t: $14.8 \mathrm{~min}$ ). $\mathrm{t}$ is the time after tadalafil was added to FaSSIF. The difference in background colour is due to different illumination settings between the runs. The orange and the green arrow illustrate an example of early and later appearing precipitate in pure FaSSIF, respectively. The blue arrow illustrates precipitate in FaSSIF with HPMC. 


\subsection{Single-particle analysis}

As described above, precipitation comprises of two processes, namely nucleation and crystal growth. The appearance of the first particle can be used as a measure for tind and hence, to assess the effect of HPMC on nucleation. This time point was measured 20 24 times for each of the HPMC concentrations (Figure 2). The tind for tadalafil in FaSSIF was $4.5 \pm 0.9$ min (Figure 2), and the addition of $0.001 \%(\mathrm{w} / \mathrm{v})$ HPMC to the dissolution medium did not significantly prolong this time (5.1 $\pm 1.5 \mathrm{~min}, \mathrm{p}>0.05)$. In contrast, with the addition of $0.01,0.1$ or $0.5 \%$ (w/v) of HPMC to the dissolution medium, the tind for tadalafil in FaSSIF was significantly prolonged compared to tadalafil in FaSSIF without HPMC at the same level of supersaturation (Figure 2). For $0.01 \%$ $(\mathrm{w} / \mathrm{v})$ HPMC, the tind was prolonged to $6.2 \pm 2.0 \mathrm{~min}$. Increasing the concentration of HPMC 10-fold further prolonged the tind, but the effect plateaued at a tind of about $9.5 \mathrm{~min}$. A HPMC concentration of $0.5 \%(\mathrm{w} / \mathrm{v})$ resulted in a tind similar to the one observed with $0.1 \%(w / v)$ HPMC present, albeit with a markedly reduced standard deviation (Figure 2).

It should be noted that the minimum detectable particle size is $0.5 \mu \mathrm{m}$ according to the technical specifications of the system, and thus the precipitation will have started already before any particles become visible. This will result in a lag time between the initial time of nucleation and the time point where the particles have reached a detectable size. However, as tind is defined here as the timepoint where the software identifies the first particle in focus, the determination was consistent for all measurements. The tind is used for comparative purposes, and not for exact modelling or extrapolation of initial nucleation, and hence, a consistent estimate is considered adequate.

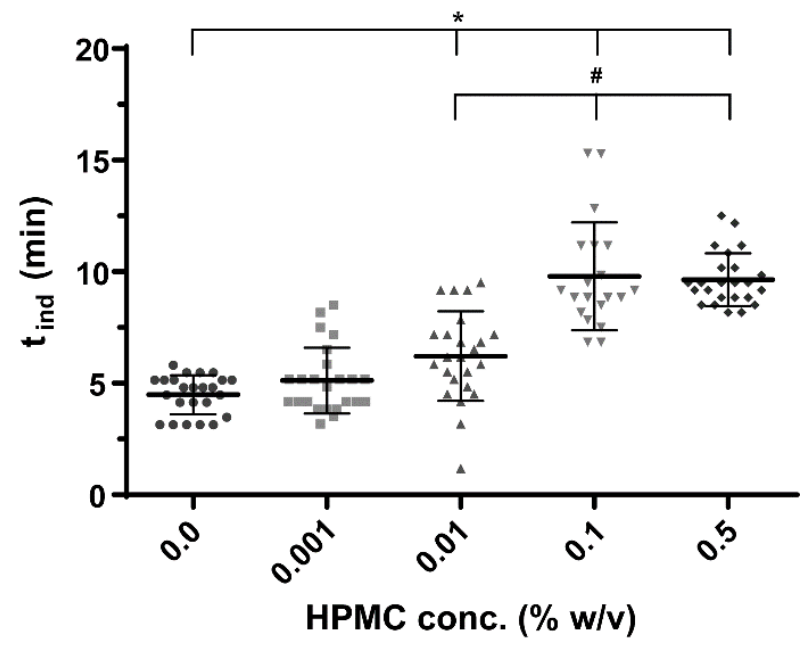

Figure 2. tind for tadalafil in FaSSIF with different concentrations of predissolved HPMC observed with the single-particle analysis method (mean $\pm S D$ ). For each concentration of HPMC the experiment was repeated 20-24 times. The * indicates a significant difference from the tind for tadalafil in FaSSIF without HPMC $(\mathrm{p}<0.05)$. The \# indicates a significant difference from the tind for tadalafil in FaSSIF with $0.01 \%(w / v)$ HPMC (p < 0.05).

The growth of tadalafil particles in FaSSIF, plotted as a function of time, with different concentrations of predissolved HPMC is shown in Figure 3. The graph for each concentration of HPMC represents the growth of a single well-defined particle from 24 replications of the experiment. For tadalafil in FaSSIF without HPMC, the first particle appears after 5 min, corresponding to the tind in Figure 2 and hereafter, a linear growth in area is observed until the end of the study (22 min). The growth rate of tadalafil was not significantly decreased in FaSSIF with $0.001 \%$ (w/v) HPMC. Maximal inhibition of the growth rate was reached with $0.01 \%(\mathrm{w} / \mathrm{v})$ HPMC, and no further decrease in the crystal growth was achieved with higher HPMC concentrations $(0.1$ and $0.5 \%$ $(\mathrm{w} / \mathrm{v}))$. In a different setup, the size of a single representative particle has previously been used to investigate dissolution phenomena. Svanbäck et al. (20) showed an excellent correlation between the dissolution measured by UV spectroscopy and the reduction in particle size followed via video-microscopy, indicating that a single particle in principle can be used to explain the behaviour of a population of particles. 


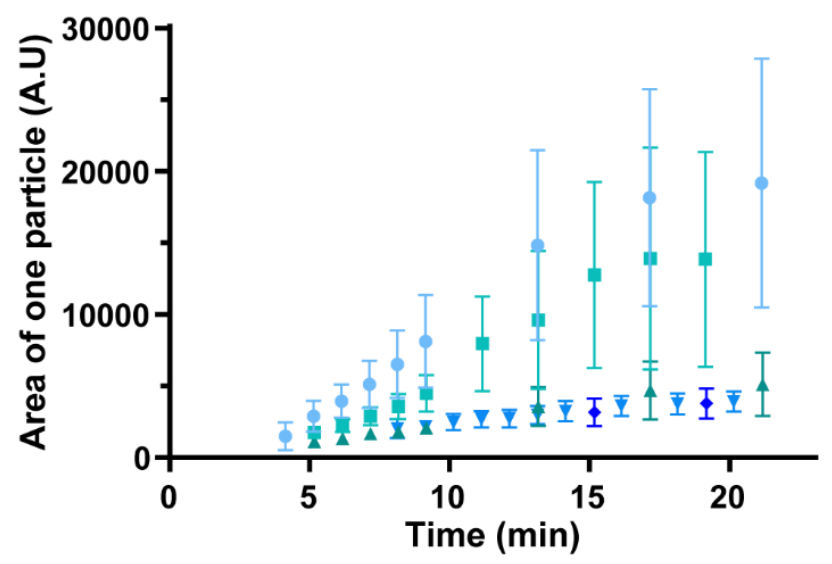

Figure 3. Area of single well-defined tadalafil particles in FaSSIF with different concentrations of predissolved HPMC as a function of time (mean \pm SD). For each of the HPMC concentrations the experiment was repeated 24 times, one well-defined particle was followed each time, and the individual area of the particle was calculated $(\mathrm{n}=3-24) .0=0.0 \%(\mathrm{w} / \mathrm{v}), \quad \square=0.001 \%(\mathrm{w} / \mathrm{v}), \mathbf{\Delta}=0.01 \%(\mathrm{w} / \mathrm{v}), \nabla=$ $0.1 \%(\mathrm{w} / \mathrm{v}), \diamond=0.5 \%(\mathrm{w} / \mathrm{v})$ HPMC.

\subsection{Multi-particle analysis}

Whilst the single-particle analysis focused on one early appearing particle, growth of only a selected particle may not be representative for the overall growth. To be able to describe the entire population of particles, and thus to include more data in the data analysis, an algorithm was developed to detect the appearing particles and calculate their areas (section 2.2.5). The induction time is defined as the first detection of a particle by the algorithm, and the same micrograph before and after segmentation is shown in Figure 4. The multi-particle analysis was able to find the majority of the particles. It is worth to note that the circular Hough transform used for particle counting was incapable of finding particles of which the geometric centers were outside the micrograph (Figure 4B). However, this was not considered an issue for the growth analysis as this was consistent and had no impact on the area measurement. 


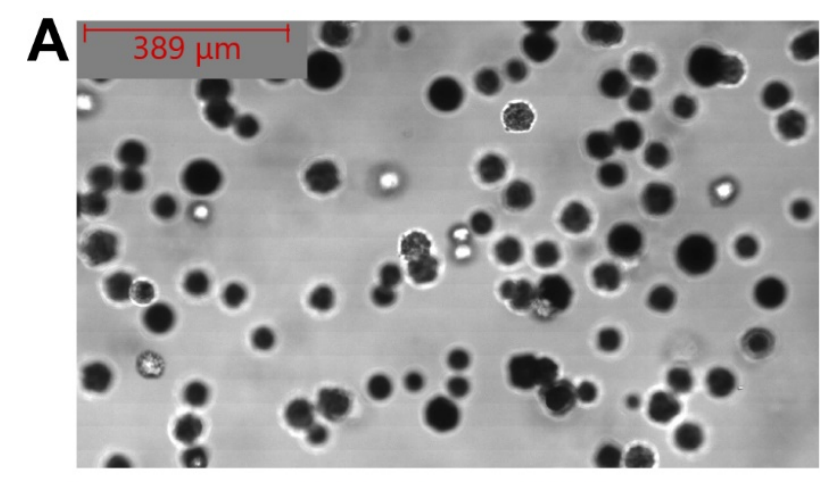

\section{B}

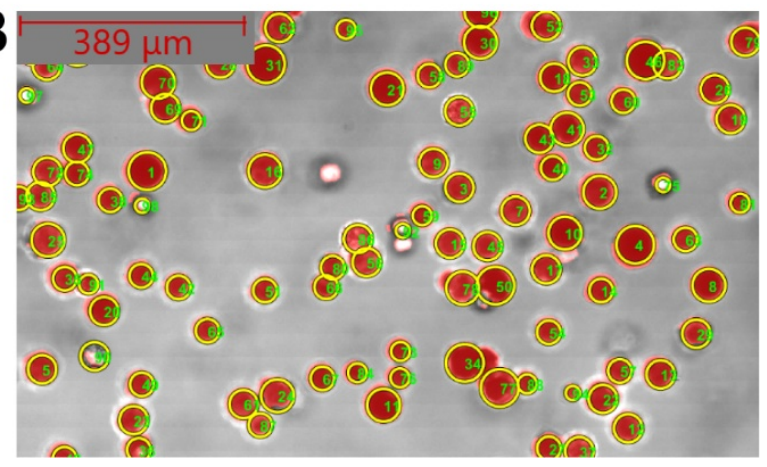

Figure 4. A: $652 \mu \mathrm{g} / \mathrm{mL}$ tadalafil in FaSSIF with $0.001 \%(\mathrm{w} / \mathrm{v})$ HPMC (t: $13.2 \mathrm{~min})$, B: the same micrograph after segmentation, where the red section describes the area of the particles and the yellow circles are used to count the particles using the multi-particle analysis.

Using this algorithm, the $t_{\text {ind }}$ for tadalafil in FaSSIF, without HPMC, was found to be $4.6 \pm 0.7$ min (Figure 5). In the presence of $0.001 \%$ (w/v) HPMC no significant difference in tind was observed (5.4 $\pm 1.1 \mathrm{~min}, \mathrm{p}<0.05)$, when compared to the tind for tadalafil in pure FaSSIF. A significantly prolonged tind was, however, observed for tadalafil in FaSSIF with 0.01 or 0.1 (w/v) HPMC $(7.1 \pm 1.8 \mathrm{~min}$ and $9.2 \pm 1.7 \mathrm{~min}$, respectively), whereas a plateau effect was observed with the addition of $0.5 \%$ (w/v) HPMC $(9.5 \pm 1.1 \mathrm{~min}, \mathrm{p}<0.05)$, as this did not further prolong the tind significantly compared to $0.1 \%$ (w/v) HPMC. These induction times were consistent with the results observed for the single-particle analysis (Figure 2). In a recent study, the effect of $\operatorname{HPMC}(0.05 \% \mathrm{w} / \mathrm{v})$ on the nucleation of tadalafil in FaSSIF was investigated by a solvent shift method in a dissolution setup (6), and these authors also found that HPMC had a pronounced effect on the nucleation of tadalafil in FaSSIF and significantly

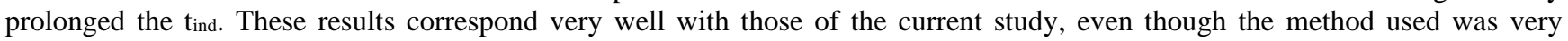
different. PI effects of HPMC have also been shown using amorphous solid dispersions as a method to induce supersaturation for tadalafil. An increase in HPMC concentration (1:1 - 1:8 w/w ratio of drug to polymer, corresponding to $0.013 \% \mathrm{w} / \mathrm{v}-0.024 \%$ w/v HPMC in the dissolution medium) resulted in an increase in the maximum dissolved drug concentration achieved during a dissolution study and a retardation of drug precipitation in water (3).

A similar plateau effect of HPMC on the tind has previously been reported for the compound RS-8359, in a dissolution setup (13), where the precipitation was inhibited by adding $0.001 \%(w / v)$ HPMC, but the effect did not further improve by addition of up to $0.1 \%(w / v)$ HPMC. 


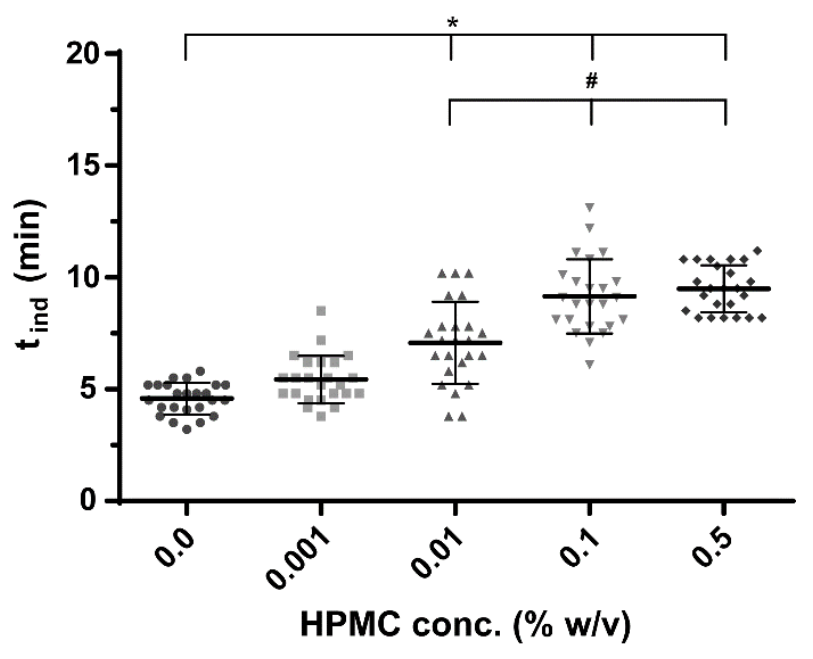

Figure 5. tind for tadalafil in FaSSIF with different concentrations of predissolved HPMC observed with the multi-particle analysis algorithm (mean \pm SD). For each concentration of HPMC the experiment was repeated 24 times. The * indicates a significant difference from the tind for tadalafil in FaSSIF without HPMC $(\mathrm{p}<0.05)$. The \# indicates a significant difference from the tind for tadalafil in FaSSIF with $0.01 \%(\mathrm{w} / \mathrm{v})$ HPMC $(\mathrm{p}<0.05)$.

The multi-particle analysis described the nucleation and subsequent crystal growth of the entire investigated area (Figure 6). Until approximately 10 min, no significant difference in the growth of tadalafil particles with and without the presence of HPMC was observed. However, after $10 \mathrm{~min}$, a clear difference was observed in the growth trend of tadalafil particles in the different investigated systems. With 0 and $0.001 \%(\mathrm{w} / \mathrm{v})$ HPMC present, a steep growth curve was observed after 10 min and continued throughout the study (22 min). With $0.01-0.5 \%$ (w/v) HPMC present, the nucleation and crystal growth of tadalafil seemed to be inhibited for approximately $13 \mathrm{~min}$, and after $15 \mathrm{~min}$ the growth of tadalafil particles increased. When analysing the area covered by precipitated tadalafil, a maximum inhibitory effect therefore was obtained already with only $0.01 \%$ (w/v) HPMC present, which was also concluded from the single-particle analysis. A maximum inhibitory effect of HPMC around $0.01 \%$ (w/v) has also been described for RS-8359 (13), but a further increase in HPMC concentration lead to faster precipitation.

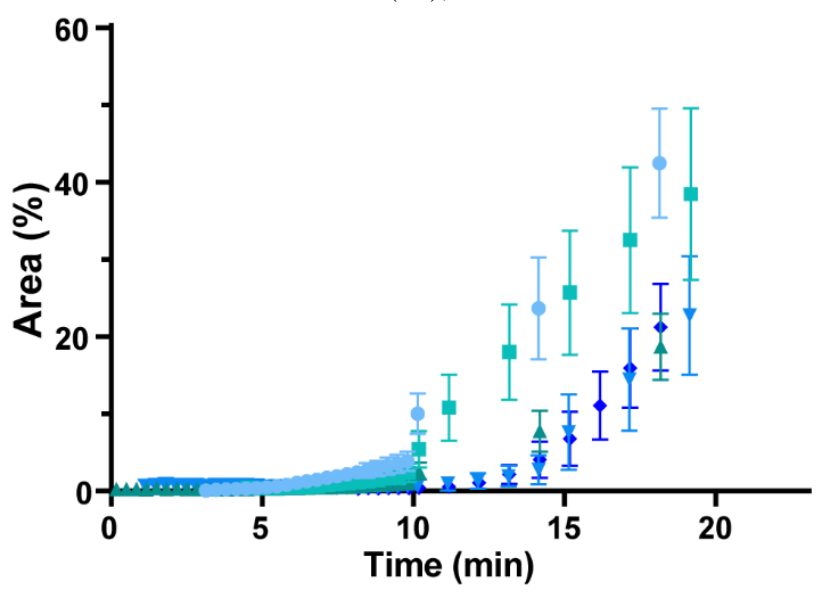

Figure 6. Percentage of the micrograph covered by tadalafil particles in FaSSIF with different concentrations of HPMC as a function of time. The growth kinetics are shown as the area (\%) of a micrograph covered by particles, calculated with the multi-particle analysis $($ mean $\pm \mathrm{SD}, \mathrm{n}=24) .0=0.0 \%(\mathrm{w} / \mathrm{v}), \mathbf{\square}=0.001 \%(\mathrm{w} / \mathrm{v}), \mathbf{\Delta}=0.01 \%(\mathrm{w} / \mathrm{v}), \nabla=0.1 \%(\mathrm{w} / \mathrm{v}), \diamond=0.5 \%(\mathrm{w} / \mathrm{v}) \mathrm{HPMC}$.

Another way to describe the particle population is to count the total number of particles, e.g. describing the number of nucleated particles. The number of tadalafil particles at three different time points, 10.0 min, 14.5 min and 17.5 min is shown in Figure 7 . 
For FaSSIF without addition of HPMC and for the lowest HPMC concentration $(0.001 \%(\mathrm{w} / \mathrm{v}))$, an increase in the particle count from 10 to $14.5 \mathrm{~min}$ was observed, but not from 14.5 to $17.5 \mathrm{~min}$. However, for $0.01 \%$ (w/v) HPMC, the rate of particle formation was constant throughout the three time points. For the high HPMC concentrations $(0.1-0.5 \%(\mathrm{w} / \mathrm{v}))$ very few particles at 10 and 14.5 min were observed however, there were significantly more particles at the last time point $(17.5 \mathrm{~min})(\mathrm{p}<0.05)$. This corresponds well to the observations in Figure 5, where the first particles appear after approximately $10 \mathrm{~min}$, for 0.1 and $0.5 \%$ (v/w) HPMC. Hence, by adding $0.01 \%(w / v)$ of HPMC to FaSSIF, it was possible to significantly inhibit both nucleation and crystal growth of tadalafil (Figure 6), however $0.1 \%$ (w/v) HPMC was needed to obtain maximal inhibitory effect of HPMC on nucleation alone (Figure 5). For felodipine, it was possible to inhibit precipitation using HPMC concentrations two times lower than the ones used in the current study (26). Hence, as described in the introduction, the optimum polymer concentration for precipitation inhibition is drug dependent.

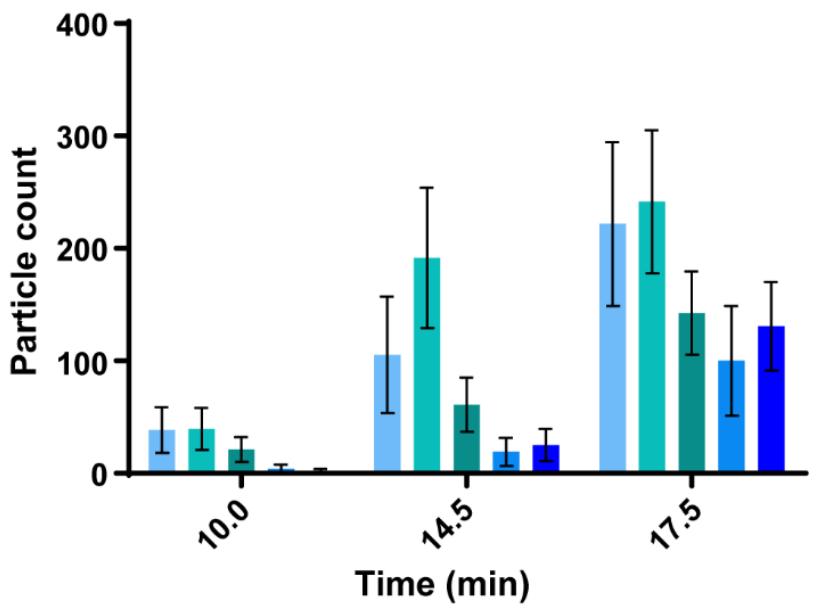

Figure 7. Histogram showing the number of particles (mean \pm SD) present at 10, 14.5 and 17.5 min ( \pm 0.5 min) for a supersaturated tadalafil suspension in FaSSIF with $0-0.5 \%(\mathrm{w} / \mathrm{v})$ HPMC $(\mathrm{n}=24)$. $=0.0 \%(\mathrm{w} / \mathrm{v}), \quad \square=0.001 \%(\mathrm{w} / \mathrm{v}), \square=0.01 \%(\mathrm{w} / \mathrm{v})$, $\square=0.1 \%$ $(\mathrm{w} / \mathrm{v}), \boldsymbol{\square}=0.5 \%(\mathrm{w} / \mathrm{v})$ HPMC.

The developed method can be used as a screening tool to evaluate the effect of a PI on supersaturation. The effect of a PI on supersaturation of a given poorly water-soluble drug is valuable information during the preformulation phase. As described in the introduction, the effect of a PI is very drug specific, and it will often be necessary to screen several PI types, concentrations and grades before deciding on the optimal supersaturating system for a given drug. Thus, the information from this work provides qualitative knowledge about the effect of a PI on supersaturation, which can be used as an efficient and highly needed screening tool for the improvement of oral absorption of poorly water-soluble drugs using supersaturating systems.

Furthermore, the micrographs can give important information about the morphology and number of particles, whilst nonmicroscopy methods, e.g. dissolution and dynamic light scattering, do not provide the same level of detail or understanding of the system. For instance, morphological differences in the precipitating particles in the presence or absence of a PI can be an indication for molecular interactions between the drug and the PI. The possibility of using 96-well plates in an automatized setup offers the potential for an effective high-throughput method where only small amounts of drug and PI are needed. Thus, this method is less time consuming and labour intensive, but yields comparable results to existing setups, e.g. conventional microscopy, where only one sample at a time can be measured $(9,19)$.

However, like any other setup, this method has its limitations. It takes the oCelloScope ${ }^{\mathrm{TM}}$ approximately 3 min to screen a 96well plate. Determination of tind may require micrographs taken more frequently, and due to the lag time, this will be an estimate for tind. Nevertheless, for comparative measurements and as an initial tool to screen polymers for their PI effect, this is not considered a problem and the developed method appears appropriate.

\subsection{Future perspective of the algorithm}

The current image analysis is optimized for spherical particles; however, drug precipitates may have other shapes, for instance, carbamazepine forms needle-like structures and algorithms to account for this type of structures could be implemented, as proposed by Wu et al. (27). This proof-of-principle study has shown that it is possible to use the oCelloScope ${ }^{\mathrm{TM}}$ as a screening tool 
of the effect of PIs in supersaturation studies. Future studies should include tracking of the particles over time which would enable conduction of single-particle analysis without any bias from manual selection. Optical flow algorithms like Farnebäck's flow algorithm (28) would probably solve this.

\section{Conclusion}

A novel small scale high-throughput method has been developed to study the effect of precipitation inhibitors on poorly-water soluble drugs in supersaturated systems. A single-particle analysis was possible using commercially available software; however, to investigate the entire population of particles an algorithm for multi-particle analysis was designed. The data output was comparable between the two methods of analysis and yielded the same ranking of the inhibitory effect on precipitation of the different HPMC concentrations. Analysis of the data showed that $0.01 \%(\mathrm{w} / \mathrm{v})$ HPMC was sufficient to delay both nucleation and crystal growth of tadalafil in FaSSIF, however $0.1 \%$ (w/v) HPMC was needed to obtain maximal inhibitory effect of HPMC on nucleation alone.

\section{Acknowledgements}

This study was conducted as part of the Oral Biopharmaceutics Tools (ORBITO) project (http://www.orbitoproject.eu), funded by the Innovative Medicines Initiative (IMI) Joint Undertaking under Grant Agreement No. 115369. Furthermore, Line Hagner Nielsen would like to thank the Danish Research Council for Technology and Production (FTP), Project DFF-4004-00120B for financial support, and in addition, the Danish National Research Foundation (project DNRF122) and Villum

Foundation's Center (Grant No. 9301) for Intelligent Drug Delivery and Sensing Using Microcontainers and Nanomechanics (IDUN) is acknowledged.

\section{Abbreviations}

1. Taylor, L. S.; Zhang, G. G. Physical Chemistry of Supersaturated Solutions and Implications for Oral 1276-1288. 
349 4. Brouwers, J.; Brewster, M. E.; Augustijns, P. Supersaturating Drug Delivery Systems: The Answer to 350 Solubility-Limited Oral Bioavailability? J. Pharm. Sci. 2009, 98(8), 2549-2572.

351 5. Gao, P.; Shi, Y. Characterization of Supersaturatable Formulations for Improved Absorption of Poorly 352 Soluble Drugs. AAPS J. 2012, 14(4), 703-713.

353 6. Palmelund, H.; Madsen, C. M.; Plum, J.; Mullertz, A.; Rades, T. Studying the Propensity of Compounds 354 to Supersaturate: A Practical and Broadly Applicable Approach. J. Pharm. Sci. 2016, 105(10), 30213553029.

356

7. Lindfors, L.; Forssen, S.; Westergren, J.; Olsson, U. Nucleation and Crystal Growth in Supersaturated 357 Solutions of a Model Drug. J. Colloid Interface Sci. 2008, 325(2), 404-413.

8. Madsen, C. M.; Boyd, B.; Rades, T.; Mullertz, A. Supersaturation of Zafirlukast in Fasted and Fed State Intestinal Media with and without Precipitation Inhibitors. Eur. J. Pharm. Sci. 2016, 91, 31-39.

9. Raghavan, S. L.; Trividic, A.; Davis, A. F.; Hadgraft, J. Crystallization of Hydrocortisone Acetate: 361

10. Grammen, C.; Plum, J.; Van Den Brande, J.; Darville, N.; Augustyns, K.; Augustijns, P.; Brouwers, J. 363 The Use of Supersaturation for the Vaginal Application of Microbicides: A Case Study With Dapivirine. J. Pharm. Sci. 2014, 103(11), 3696-3703.

11. Bevernage, J.; Forier, T.; Brouwers, J.; Tack, J.; Annaert, P.; Augustijns, P. Excipient-Mediated Supersaturation Stabilization in Human Intestinal Fluids. Mol. Pharmaceutics. 2011, 8(2), 564-570.

12. Warren, D. B.; Bergstrom, C. A.; Benameur, H.; Porter, C. J.; Pouton, C. W. Evaluation of the Structural 368 Determinants of Polymeric Precipitation Inhibitors Using Solvent Shift Methods and Principle Component Analysis. Mol. Pharmaceutics. 2013, 10(8), 2823-2848.

13. Usui, F.; Maeda, K.; Kusai, A.; Nishimura, K.; Keiji, Y. Inhibitory Effects of Water-Soluble Polymers on Precipitation of RS-8359. Int. J. Pharm. 1997, 154(1), 59-66.

14. Raghavan, S. L.; Kiepfer, B.; Davis, A. F.; Kazarian, S. G.; Hadgraft, J. Membrane Transport of 373 Hydrocortisone Acetate from Supersaturated Solutions; The Role of Polymers. Int. J. Pharm. 2001, 221(1-2), 95-105. 
15. Patel, D. D.; Anderson, B. D. Effect of Precipitation Inhibitors on Indomethacin Supersaturation Maintenance: Mechanisms and Modeling. Mol. Pharmaceutics. 2014, 11(5), 1489-1499.

16. Hsieh, Y. L.; Box, K.; Taylor, L. S. Assessing the Impact of Polymers on the pH-Induced Precipitation Behavior of Poorly Water Soluble Compounds Using Synchrotron Wide Angle X-Ray Scattering. J. Pharm. Sci. 2014, 103(9), 2724-2735.

17. Ilevbare, G.; Taylor, L. Liquid-Liquid Phase Separation in Highly Supersaturated Aqueous Solutions of Poorly Water-Soluble Drugs: Implications for Solubility Enhancing Formulations. Cryst. Growth Des. 2013, 13(4), 1497-1509.

18. Bevernage, J.; Brouwers, J.; Brewster, M. E.; Augustijns, P. Evaluation of Gastrointestinal Drug Supersaturation and Precipitation: Strategies and Issues. Int. J. Pharm. 2013, 453(1), 25-35.

19. Wu, J. X.; Xia, D.; van den Berg, F.; Amigo, J. M.; Rades, T.; Yang, M.; Rantanen, J. A Novel Image Analysis Methodology for Online Monitoring of Nucleation and Crystal Growth During Solid State Phase Transformations. Int. J. Pharm. 2012, 433(1-2), 60-70.

20. Svanback, S.; Ehlers, H.; Yliruusi, J. Optical Microscopy As a Comparative Analytical Technique for Single-Particle Dissolution Studies. Int. J. Pharm. 2014, 469(1), 10-16.

21. Fredborg, M.; Andersen, K. R.; Jorgensen, E.; Droce, A.; Olesen, T.; Jensen, B. B.; Rosenvinge, F. S.; Sondergaard, T. E. Real-Time Optical Antimicrobial Susceptibility Testing. J. Clin. Microbiol. 2013, 51(7), 2047-2053.

22. Kloefer, B.; van Hoogevest, P.; Moloney, R.; Martin Kuentz; Mathew L.S. Leigh, a. J. D. Study of a Standardized Teurocholate-Lecithin Powder for Preparing the Biorelevant Media FeSSIF and FaSSIF. Dissolution Technol. 2010, 6-13.

23. Shin-Etsu Chemical Co., Ltd. Molecular Weight and Degree of Polymerization. 2004 June.

24. Soille, P. Morphological Image Analysis: Principles and Applications. 2nd ed.; Springer-Verlag Berlin Heidelberg, 1999.

25. Davies, E. R. A Modified Hough Scheme for General Circle Location. Pattern Recognit. Lett. 1988, 7(1), 37-43. 
401 26. Alonzo, D. E.; Raina, S.; Zhou, D.; Gao, Y.; Zhang, G. G. Z.; Taylor, L. S. Characterizing the Impact of 402 Hydroxypropylmethyl Cellulose on the Growth and Nucleation Kinetics of Felodipine from 403 Supersaturated Solutions. Cryst. Growth Des. 2012, 12(3), 1538-1547.

404 27. Wu, J. X.; Kucheryavskiy, S. V.; Jensen, L. G.; Rades, T.; Müllertz, A.; Rantanen, J. Image Analytical 405 Approach for Needle-Shaped Crystal Counting and Length Estimation. Cryst. Growth Des. 2015, 15(10), $406 \quad 4876-4885$.

407 28. Farnebäck, G. Two-frame motion estimation based on polynomial expansion. Bigun J, Gustavsson T, 408 Eds.; Image Analysis: 13th Scandinavian Conference, SCIA 2003 Halmstad, Sweden, June 29 - July 2, 2003 Proceedings. Springer-Verlag Berlin Heidelberg, 2003; pp 363-370.

410 